ISSN 0103-9954

\title{
VERMICULITA COMO SUBSTRATO PARA O TESTE DE GERMINAÇÃO DE SEMENTES DE BARBATIMÃO
}

\author{
VERMICULITE AS SUBSTRATE FOR GERMINATION OF 'BARBATIMÃO’ SEEDS
}

\author{
Cibele Chalita Martins ${ }^{1}$ Carla Gomes Machado ${ }^{2}$ \\ Isliana Griebler Ribeiro Caldas ${ }^{3}$ Israel Gomes Vieira ${ }^{4}$
}

\begin{abstract}
RESUMO
O objetivo deste trabalho foi avaliar o desempenho da vermiculita como substrato para o teste de germinação e estabelecer qual granulometria e intensidade de umedecimento deste substrato seria mais favorável à germinação de sementes de barbatimão. A semeadura foi realizada com quatro repetições de 50 sementes (escarificadas com $\mathrm{H}_{2} \mathrm{SO}_{4} / 60 \mathrm{~min}$ ) em rolo de papel (RP) umedecido com 2,5 vezes a massa em água (testemunha) e vermiculita nas granulometrias mícron (MI), superfina (SF), fina (F) e média (M) umedecida com 0,$5 ; 1,0 ; 1,5$ e 2,0 vezes a sua massa em água. $\mathrm{O}$ teste de germinação foi conduzido a $30^{\circ} \mathrm{C}$, avaliandose a primeira e a última contagens aos 7 e 28 dias após a semeadura realizando-se avaliações semanais das plântulas normais. Foi calculado o tempo médio e a frequência relativa de germinação. $O$ teste de germinação de sementes de barbatimão pôde ser realizado em vermiculita $\mathrm{F}$ (umedecida com 2,0 vezes a sua massa em água) ou $\mathrm{M}$ (umedecida com 0,5 até 2,0 vezes a sua massa em água) por possibilitarem a máxima germinação em menor tempo (21 dias), de modo similar ao RP.
\end{abstract}

Palavras-chave: espécie florestal; umedecimento; plântulas; velocidade de germinação.

\begin{abstract}
The objective of this research was to evaluate the use of vermiculite as a substrate for the germination test of 'barbatimão' seeds and to establish what would be the best granulometry and the best moisture intensity to germinate 'barbatimão' seeds. Four replications of 50 seeds were previously scarified with $\mathrm{H}_{2} \mathrm{SO}_{4}$ for 60 minutes. Afterwards, the following germination substrates were evaluated: paper rolls moistened with water equivalent to 2.5 times the weight of the dry paper (control); micron, super thin, thin and average sized vermiculite moistened with $0.5,1.0,1.5$ and 2.0 times the weight of the dry paper. The germination temperature was $30^{\circ} \mathrm{C}$. The percentage of normal seedlings was obtained seven days after sowing (first count) and, then, weekly (up to 28 days). Average time and relative distribution of germination were calculated. It was concluded that the germination of 'barbatimão' seeds can be carried out with either fine vermiculite moistened with water equivalent to 2.0 times the weight of the dry paper or average sized substrate moistened with water equivalent to 0.5 to 2.0 times the weight of dry paper. These treatments resulted in higher and faster germination (21 days), similarly to the paper roll method.
\end{abstract}

Keywords: forest species; moistening; seedlings; speed of germination.

1. Engenheira Agrônoma, Dra ${ }^{\text {. }}$, Professora Associada, Faculdade de Tecnologia do Estado de São Paulo, Curso Superior de Tecnologia em Silvicultura, Rua Amantino de Oliveira Ramos, 60, CEP 18300-000, Capão Bonito (SP). cibele@fca.unesp.br

2. Engenheira Agrônoma, Drª ${ }^{\text {. }}$ Professora da Universidade Estadual de Goiás, Unidade Universitária de Palmeiras de Goiás, Rua São João, s/n, CEP 76190-000, Palmeiras de Goiás (GO). carlagomesmachado@gmail.com

3. Engenheira Florestal, Mestranda do curso de Engenharia Florestal, Universidade Estadual Paulista, Caixa Postal 237, CEP 18610-307, Botucatu (SP). igrcaldas@fca.unesp.br

4. Biólogo, MSc., Pesquisador do Instituto de Pesquisas e Estudos Florestais, Escola Superior de Agricultura Luiz de Queiroz, Universidade de São Paulo, Avenida Pádua Dias, 11, Caixa Postal 530, CEP 13400-970, Piracicaba (SP). ipef@ipef.br

Recebido para publicação em 14/08/2009 e aceito em 21/09/2010 


\section{INTRODUÇÃO}

O barbatimão (Stryphnodendron adstringens (Mart.) Coville) é uma espécie arbórea nativa dos cerrados brasileiros que costuma ser utilizada para a extração de madeira e na medicina popular devido à casca com alto teor de tanino. $\mathrm{O}$ extrativismo desordenado da planta é exercido pela população e vem provocando esgotamento deste recurso. Assim, é uma espécie que necessita de diretrizes para a sua conservação, desenvolvimento de técnicas de manejo sustentado e programas de domesticação e recomposição de matas nativas (BORGES FILHO e FELFILI, 2003; MARTINS et al., 2008).

O uso de sementes de qualidade é de grande importância para a propagação e produção de mudas e o teste de germinação é o principal parâmetro de avaliação da qualidade fisiológica das sementes. O resultado deste teste é utilizado para a determinação da taxa de semeadura, comparação do valor de lotes diferentes e comercialização, pois possibilita a obtenção de resultados comparáveis entre laboratórios (CARVALHO e NAKAGAWA, 2000).

Para a quase totalidade das sementes de espécies florestais os procedimentos de germinação ainda não estão padronizados pelas Regras para Análise de Sementes - RAS (BRASIL, 2009), publicação que normatiza a metodologia deste teste, quanto às recomendações de temperaturas, tipos e intensidade de umedecimento dos substratos, superação de dormência, critérios de avaliação, entre outras. Essas recomendações visam oferecer condições supostamente ideais ao teste para estimar o potencial máximo de germinação de um lote. As RAS (BRASIL, 2009) são corrigidas e atualizadas periodicamente e as alterações são realizadas com base em pesquisas científicas.

O substrato influencia a germinação, em função de sua estrutura, aeração, capacidade de retenção de água, propensão à infestação por patógenos, dentre outros, podendo favorecer ou prejudicar a germinação das sementes. Constitui o suporte físico no qual a semente é colocada e tem a função de manter as condições adequadas para a germinação e o desenvolvimento das plântulas (FIGLIOLIA et al., 1993). Portanto, o tipo de substrato utilizado deve ser adequado às exigências fisiológicas de germinação de cada espécie, tamanho e forma da semente (BRASIL, 2009).

$\mathrm{O}$ umedecimento do teste de germinação deve ocorrer em níveis de água adequados ao tipo de substrato a ser utilizado, pois materiais diferentes possuem características próprias de porosidade, agregação, superfície de contato com a semente e capacidade de retenção de água. Não havendo disponibilidade hídrica suficiente, o processo de germinação pode ser seriamente prejudicado, podendo ocorrer a morte do embrião (MARCOS FILHO, 2005). De forma oposta, o excesso de água no substrato pode prejudicar o processo germinativo, devido à menor aeração (MARCOS FILHO, 2005). Estes fatos são comprovados por trabalhos sobre o umedecimento na germinação, realizados com sementes de várias espécies, florestais e agrícolas (PHANEENDRANAT, 1980; TAO, 1981; TOLEDO; PEDREIRA, 1984; VARELA et al., 2005; RAMOS et al., 2006, MARTINS et al., 2009).

Devido à importância da utilização da quantidade certa de água para a germinação, as Regras para Análise de Sementes (BRASIL, 2009) recomendam para o teste de germinação em papel, a adição de um volume de água equivalente a 2,0 até 2,5 vezes (gramínea) ou 2,5 a 3,0 vezes (leguminosa) à massa do substrato. Para areia é recomendado o umedecimento com até $50 \%$ e $60 \%$ da capacidade de retenção do substrato areia em água, para sementes de cereais e de leguminosas, respectivamente; por sua vez para o substrato vermiculita não há recomendações.

A vermiculita é um substrato comumente utilizado para a produção de mudas de espécies florestais e também poderia ser utilizado nos laboratórios de análise de sementes como substrato para o teste padrão de germinação, devido às vantagens como: fácil obtenção, uniformidade na composição química e granulométrica, porosidade, capacidade de retenção de água e baixa densidade (FIGLIOLIA et al., 1993; MARTINS et al., 2009). Adicionalmente, é um produto estéril, devido ao processo de expansão que é realizado entre $800 \mathrm{e}$ $900^{\circ} \mathrm{C}$ (ISOLANTES, 2009). Quanto à granulometria do material, existem para a comercialização quatro tipos de vermiculita: mícron (90 a $100 \%$ das partículas entre $0,15-0,20 \mathrm{~mm}$ ), superfina (95 a $100 \%$ das partículas entre $0,21-0,30 \mathrm{~mm})$, fina (90 a $100 \%$ das partículas entre $0,30-0,50 \mathrm{~mm}$ ) e média (90 a 100\% das partículas entre 1,19-0,50mm).

Existem relatos que o teste de germinação de sementes de Stryphnodendron adstringens deve ser realizado em substrato papel, com sementes submetidas a tratamento de superação de dormência, e nas temperaturas constantes de 25,30 ou $35^{\circ} \mathrm{C}$ ou

Ci. Fl., v. 21, n. 3, jul.-set., 2011 
alternadas de $20-30^{\circ} \mathrm{C}$ (MARTINS et al., 2008).

$\mathrm{O}$ objetivo deste trabalho foi avaliar o desempenho da vermiculita como substrato para o teste de germinação e estabelecer qual granulometria e intensidade de umedecimento deste substrato seria mais favorável à germinação de sementes de barbatimão.

\section{MATERIAL E MÉTODOS}

As sementes de Stryphnodendron adstringens originaram-se de 20 plantas localizadas na região de Botucatu, Estado de São Paulo, as quais foram extraídas manualmente das vagens e submetidas à limpeza para a retirada de sementes chochas, mal formadas e danificadas por fungos e insetos, originando um lote de sementes.

No Laboratório de Análise de Sementes do Departamento de Produção Vegetal da Faculdade de Ciências Agronômicas da UNESP, as sementes foram escarificadas com ácido sulfúrico por 60 minutos para a superação da dormência (MARTINS et al., 2008) e semeadas nos seguintes substratos: rolo de papel (testemunha) e entre vermiculita mícron, superfina, fina e média.

O umedecimento do substrato papel foi realizado com 2,5 vezes a massa deste em água (BRASIL, 2009) e o substrato vermiculita com $0,51,01,5$ e 2,0 vezes a sua massa em água. Não houve reumedecimento dos substratos durante o decorrer do experimento. Para que a distância entre as sementes em ambos os tipos de substratos (papel e vermiculita) fosse igual, a germinação em vermiculita foi conduzida em caixas plásticas de $30,2 \mathrm{~cm} \times 20,8 \mathrm{~cm} \times 6,3 \mathrm{~cm}$ por serem similares em comprimento e largura às dimensões do papel e, com exceção desse último substrato, as sementes foram semeadas a $5 \mathrm{~mm}$ de profundidade.

Nascâmarasdegerminação, após ainstalação do teste, os rolos de papel e as caixas plásticas com substrato permaneceram acondicionados dentro de sacos plásticos de $0,033 \mathrm{~mm}$ de espessura fechados, para evitar a desidratação (COIMBRA et al., 2007).

$\mathrm{O}$ efeito dos substratos sobre o desempenho germinativo das sementes foi avaliado pelo teste de germinação e de primeira contagem de germinação instalado com quatro repetições de 50 sementes, colocadas para germinar na temperatura constante de $30^{\circ} \mathrm{C}$ (MARTINS et al., 2008). A avaliação do teste de primeira contagem foi realizada 7 dias após a semeadura, contabilizando-se a porcentagem de plântulas normais. A partir dessa data, a contagem de plântulas normais foi realizada semanalmente até que a germinação cessasse (28 dias) quando foram calculadas as porcentagens de germinação (plântulas normais), plântulas anormais, sementes mortas e dormentes (duras).

No procedimento estatístico, a análise de variância foi realizada separadamente para cada teste, segundo delineamento inteiramente casualizado com quatro repetições, com comparação entre as médias dos tratamentos por meio do teste de Tukey, ao nível de 5\% de probabilidade. Antes das análises, os dados de plântulas anormais foram transformados em $(x+0,5)^{1 / 2}$ e, de sementes mortas, em arco-seno $(\mathrm{x} / 100)^{1 / 2}$ (BANZATTO e KRONKA, 2006). Os demais parâmetros não necessitaram de transformação. As médias apresentadas nas tabelas são de dados não transformados. A partir do número de sementes germinadas, foi calculado o tempo médio e a frequência relativa de germinação (SANTANA e RANAL, 2004).

\section{RESULTADOS E DISCUSSÃO}

No teste de germinação não foram encontradas sementes dormentes, pois o tratamento de superação de dormência utilizado foi eficiente. Assim, esses dados não foram apresentados por terem sido nulos. As sementes de barbatimão apresentaram porcentagens de germinação e de sementes mortas estatisticamente similares em todos os substratos e intensidades de umedecimento avaliado (Tabela 1).

Esses resultados permitem inferir que as sementes de barbatimão apresentam uma boa plasticidade fenotípica quanto às exigências de substrato e umedecimento para a germinação, quando comparadas às de outras espécies, estando adaptadas para germinar tanto em rolo de papel como em vermiculita de diversas granulometrias, sob amplas condições de umedecimento (PHANEENDRANAT, 1980; TAO, 1981; TOLEDO e PEDREIRA, 1984; NASCIMENTO et al., 2003; VARELA et al., 2005; LIMA et al., 2006; RAMOS et al., 2006, MARTINS et al., 2009). Essas características são adaptações evolutivas que conferem maior agressividade em relação a outras plantas na ocupação de um ecossistema (ODUM, 2001).

Nos gráficos de frequência relativa (Figuras 1 e 2) pode-se verificar que, em todos os tratamentos, as sementes de barbatimão germinaram até atingir um valor máximo no $7^{\circ}$ dia após a semeadura e esse valor declinou posteriormente. 
No entanto, existiram condições que permitiram que as sementes germinassem mais rapidamente, garantindo a expressão do seu potencial fisiológico máximo, como recomendam as Regras para Análise de Sementes para este teste (BRASIL, 2009).

No teste da primeira contagem, os tratamentos que apresentaram sementes com germinação mais rápida foram: a semeadura em rolo de papel (RP), vermiculita mícron (MI) umedecida com 1,0 vez a sua massa em água, vermiculita superfina (SF) umedecida com quantidades de 1,0 até 2,0 vezes a sua massa em água e vermiculita fina (F) ou média (M) em todas as condições de umedecimento avaliadas (Tabela 1). No entanto, somente alguns desses tratamentos, tais como a semeadura em rolo de papel, vermiculita superfina umedecida com 1,0 ou 2,0 vezes a sua massa em água ( $\mathrm{SF} 1,0$ e 2,0), vermiculita fina umedecida com 2,0 vezes a sua massa em água $(F 2,0)$ ou média em todas as condições de umedecimento avaliadas
(M 0,$5 ; 1,0 ; 1,5$ e 2,0) possibilitaram também um menor tempo médio de germinação, entre 7,7 e 8,5 dias (Figuras 1 e 2).

Assim, devido à maior velocidade de germinação, nos tratamentos rolo de papel (testemunha), vermiculita fina umedecida com 2,0 vezes a massa em água (F 2,0) e vermiculita média umedecida com quaisquer das quantidades de água (M 0,5;1,0;1,5 e 2,0), as sementes deixaram de germinar a partir do $21^{\circ}$ dia da semeadura, antecipando em uma semana a conclusão do teste de germinação, pois os demais tratamentos necessitaram de 28 dias para isso (Figuras 1 e 2).

Esses resultados confirmam resultados de Martins et al. (2008) sobre um desempenho germinativo ótimo das sementes de barbatimão em rolo de papel, mas mostram que a semeadura em vermiculita pode ser igualmente favorável, desde que esta possua a granulometria e o umedecimento ideais para a espécie.

TABELA 1: Efeito do substrato papel (RP) umedecido com 2,5 a sua massa em água (testemunha) e vermiculita mícron (MI), superfina (SF), fina (F) e média (M), umedecidas com 0,$5 ; 1,0 ; 1,5 \mathrm{e}$ 2,0 vezes a sua massa em água sobre o desempenho germinativo de sementes de barbatimão.

TABLE 1: Effect of the paper substract (RP) moistened with 2.5 of its mass in water (witness) and micron vermiculite (MI), super thin (SF), thin (F) and medium (M), moistened with $0.5 ; 1.0$; 1.5 and 2.0 times its mass in water over the germinating performance of 'barbatimão' seeds.

\begin{tabular}{|c|c|c|c|c|c|}
\hline Substrato & $\begin{array}{c}\text { Vezes a massa } \\
\text { do substrato } \\
\text { em água }\end{array}$ & $\begin{array}{l}\text { Primeira } \\
\text { Contagem } \\
(\%)\end{array}$ & Germinação (\%) & $\begin{array}{c}\text { Plântulas } \\
\text { Anormais } \\
(\%)^{1}\end{array}$ & $\begin{array}{c}\text { Sementes } \\
\text { Mortas } \\
(\%)^{2}\end{array}$ \\
\hline RP (testemunha) & 2,5 & $64 \mathrm{abc}$ & $71 \mathrm{a}$ & $7 \mathrm{a}$ & $22 \mathrm{a}$ \\
\hline \multirow[t]{4}{*}{ MI } & 0,5 & 33 & $77 \mathrm{a}$ & $6 \mathrm{a}$ & $17 \mathrm{a}$ \\
\hline & 1,0 & 57 abcd & 80 a & $2 a b$ & $18 \mathrm{a}$ \\
\hline & 1,5 & $48 \quad$ cde & 81 a & $4 \mathrm{ab}$ & $15 \mathrm{a}$ \\
\hline & 2,0 & 43 & $75 \mathrm{a}$ & $5 \mathrm{ab}$ & $20 \mathrm{a}$ \\
\hline \multirow[t]{4}{*}{ SF } & 0,5 & $53 \mathrm{bcd}$ & 79 a & $2 a b$ & $19 \mathrm{a}$ \\
\hline & 1,0 & $68 \mathrm{ab}$ & $81 \mathrm{a}$ & $0 \quad b$ & 19 a \\
\hline & 1,5 & $68 \mathrm{ab}$ & $82 \mathrm{a}$ & $0 \quad b$ & $18 \mathrm{a}$ \\
\hline & 2,0 & $68 \mathrm{ab}$ & 80 a & $0 \quad b$ & $20 \mathrm{a}$ \\
\hline \multirow[t]{4}{*}{$\mathrm{F}$} & 0,5 & 58 abcd & $80 \mathrm{a}$ & $2 a b$ & $18 \mathrm{a}$ \\
\hline & 1,0 & $66 \mathrm{ab}$ & $80 \mathrm{a}$ & $3 a b$ & $17 \mathrm{a}$ \\
\hline & 1,5 & $64 \mathrm{abc}$ & $81 \mathrm{a}$ & $4 \mathrm{ab}$ & $15 \mathrm{a}$ \\
\hline & 2,0 & $67 \mathrm{ab}$ & $79 \mathrm{a}$ & $3 \mathrm{ab}$ & $18 \mathrm{a}$ \\
\hline \multirow[t]{5}{*}{ M } & 0,5 & $69 \mathrm{ab}$ & $79 \mathrm{a}$ & $4 \mathrm{ab}$ & $17 \mathrm{a}$ \\
\hline & 1,0 & $68 \mathrm{ab}$ & $80 \mathrm{a}$ & $5 \mathrm{ab}$ & $15 \mathrm{a}$ \\
\hline & 1,5 & $71 \mathrm{a}$ & $84 \mathrm{a}$ & $3 a b$ & $13 \mathrm{a}$ \\
\hline & 2,0 & $67 \mathrm{ab}$ & 73 a & $4 a b$ & $23 \mathrm{a}$ \\
\hline & & 11,2 & 8,1 & 31,12 & 20,8 \\
\hline
\end{tabular}

Em que: ${ }^{1}$ Os dados foram transformados em arc sen $((\mathrm{x}+1) / 100)^{1 / 2} ;{ }^{2} \mathrm{Os}$ dados foram transformados em arc sen $(\mathrm{x} / 100)^{1 / 2}$. Para cada característica avaliada, médias seguidas pela mesma letra na coluna não diferem estatisticamente entre si pelo teste de Tukey $(\mathrm{p}<0,05)$. As médias apresentadas são dos dados originais. 

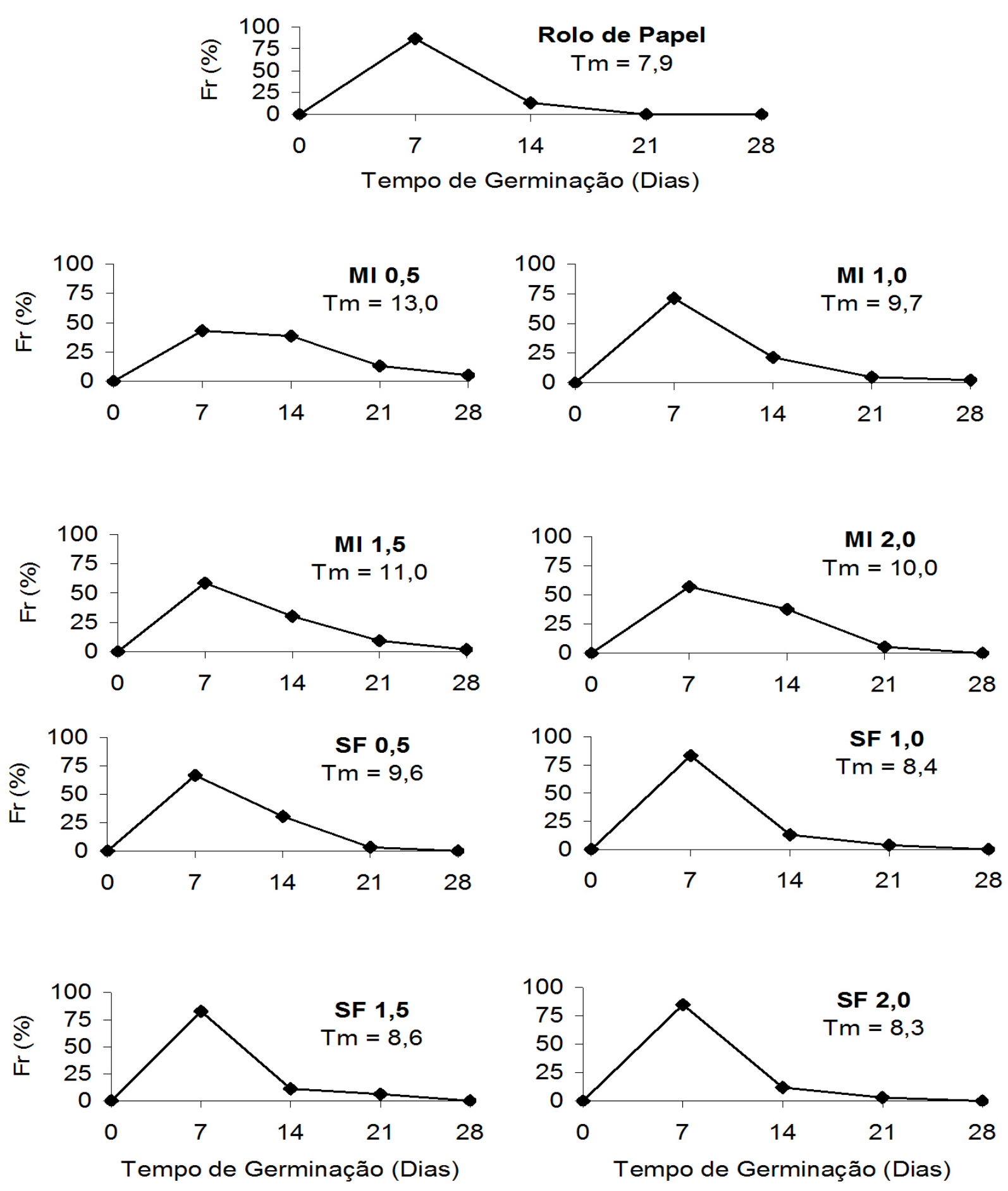

FIGURA 1: Frequência relativa (Fr) e tempo médio (Tm) da germinação de sementes de barbatimão em rolo de papel (testemunha), vermiculita mícron (MI) e superfina (SF) umedecidas com 0,5 ; 1,$0 ; 1,5$ e 2,0 vezes a sua massa em água.

FIGURE 1: Average time (Tm) and relative rate (Fr) of germination of 'barbatimão' seeds in paper roll (witness), micron vermiculite (MI) and super thin (SF) moistened with $0.5 ; 1.0 ; 1.5$ and 2.0 times its mass in water. 

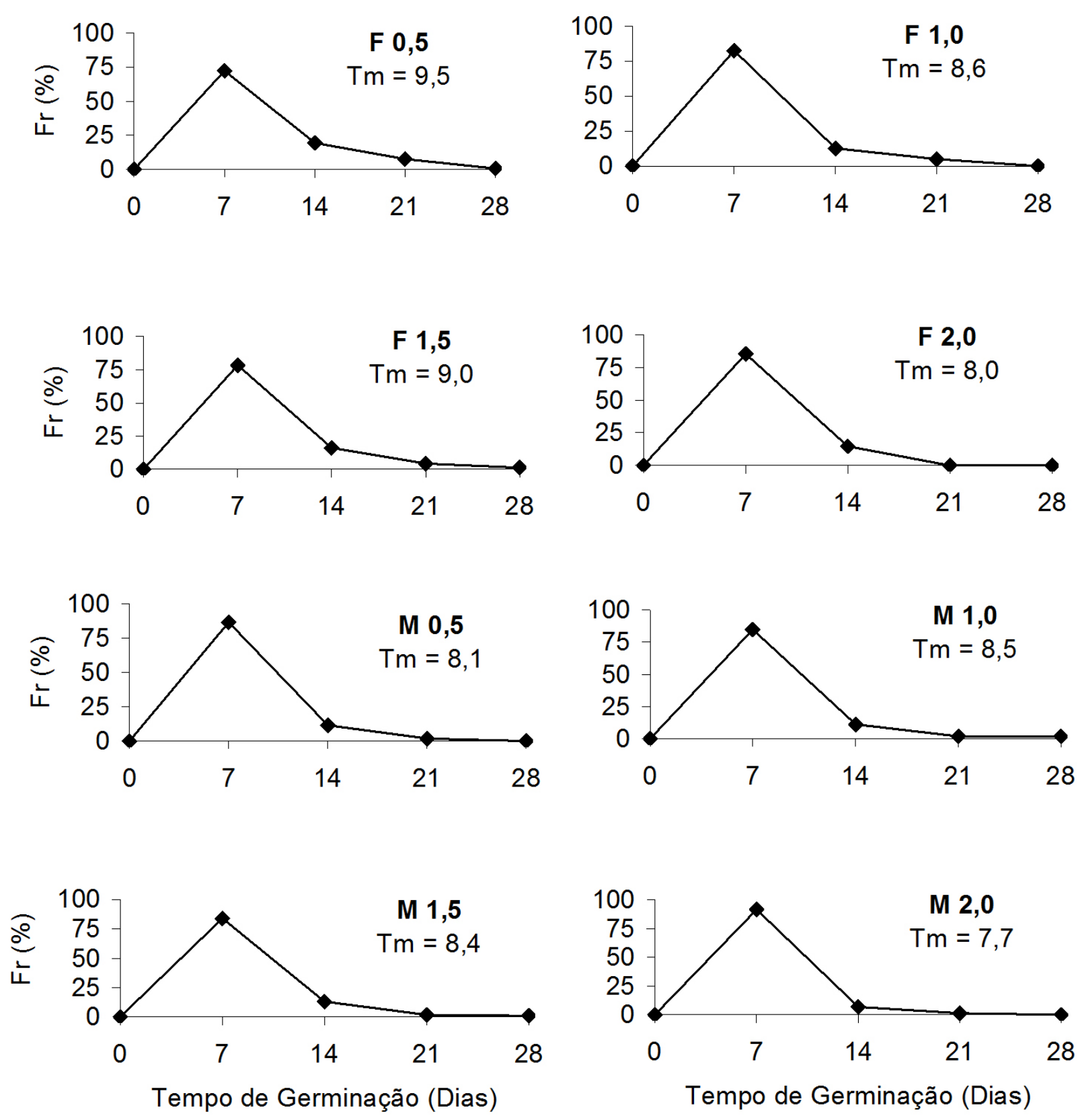

FIGURA 2: Frequência relativa (Fr) e tempo médio $(\mathrm{Tm})$ da germinação de sementes de barbatimão em vermiculita fina (F) e média (M) umedecidas com 0,$5 ; 1,0 ; 1,5$ e 2,0 vezes a sua massa em água.

FIGURE 2: Average time (Tm) and relative rate Fr) of germination of 'barbatimão' seeds in thin vermiculite (F) and medium vermiculite $(\mathrm{M})$ moistened with $0.5 ; 1.0 ; 1.5$ and 2.0 times its mass in water. 


\section{CONCLUSÕES}

O teste de germinação de sementes de barbatimão pode ser realizado em vermiculita fina (umedecida com 2,0 vezes a massa em água) ou vermiculita média (umedecida com 0,5 até 2,0 vezes a massa em água) por possibilitarem a máxima germinação em menor tempo (21 dias), de modo similar ao rolo de papel.

\section{REFERÊNCIAS BIBLIOGRÁFICAS}

BANZATTO, D. A.; KRONKA, S. N. Experimentação Agrícola. 4. ed. Jaboticabal: FUNEP, 2006. $237 \mathrm{p}$.

BORGES FILHO, H. C.; FELFILI, J. M. Avaliação dos níveis de extrativismo da casca de barbatimão (Stryphnodendron adstringens (Mart.) Coville) no Distrito Federal, Brasil. Revista Árvore, Viçosa, v. 27, n. 5, p. 735 - 745, 2003.

BRASIL. Ministério da Agricultura, Pecuária e Abastecimento. Secretaria de Defesa Agropecuária. Regras para análise de sementes. Brasília, 2009. $399 \mathrm{p}$.

CARVALHO, N. M.; NAKAGAWA, J. Sementes: Ciência, Tecnologia e Produção. 4. ed. Jaboticabal: FUNEP, 2000. $588 \mathrm{p}$.

COIMBRA, R. A., et al. Teste de germinação com acondicionamento dos rolos de papel em sacos plásticos visando a otimização dos resultados. Revista Brasileira de Sementes, Brasília, v. 29, n.1, p. 92-97, 2007.

FIGLIOLIA, M. B., et al. Análise de sementes. In: AGUIAR, I. B., et al. Sementes Florestais Tropicais. Brasília: ABRATES, 1993. p. 137-174. ISOLANTES, Condicionadores de Solo e Substratos. Minério de Vermiculita Crua Concentrada. Disponível em: (http://www.eucatex.com.br/ eucatex/descricao.asp?B2 $=\& \mathrm{~A} 1=15 \& \mathrm{~A} 2=104$ ). Acesso em: 23 de março de 2009.

LIMA, J. D. et al. Efeito da temperatura e do substrato na germinação de sementes de Caesalpinia ferrea Mart. ex Tul. (Leguminosae, Caesalpinoideae). Revista Árvore, Viçosa, v. 30, n. 4, p. 513 - 518, 2006.

MARCOS-FILHO, J. Fisiologia de Sementes de Plantas Cultivadas. Piracicaba: FEALQ,
2005. 495 p.

MARTINS, C. C. et al. Umedecimento do substrato na emergência e vigor de plântulas de pupunheira. Revista Brasileira de Fruticultura, Jaboticabal, v. 31, n. 1, p. 224 - 230, 2009.

MARTINS, C. C. et al. Temperatura e substrato para o teste de germinação de sementes de barbatimão ((Stryphnodendron adstringens (Mart.) Coville (Leguminosae)). Revista Árvore, Viçosa, v. 32, n. 4, p. 633-639, 2008.

NASCIMENTO, W. M. O. do et al. Temperatura e substrato para germinação de sementes de Parkia platycephala Benth. (Leguminosae-Caesalpinoideae). Revista de Agricultura Tropical, Cuiabá, v. 7, n. 1, p. 119129, 2003.

ODUM, E. P. Fundamentos de Ecologia. Lisboa: Fundação Calouste Gulbenkian, 2001. 979 p.

PHANEENDRANAT, B. R. Influence of amount of water in paper towel on stand germination tests. Journal of Seed Technology, v. 5, n. 2, p. 82-87, 1980.

RAMOS, M. B. P. et al. Influência da temperatura e da quantidade de água no substrato sobre a germinação de sementes de Ochroma pyramidale (Cav. ex Lam.) Urban (pau-de-balsa). Acta Amazônica, Manaus, v. 36, n. 1, p. 103-106, 2006.

SANTANA, D. G.; RANAL, M. A. Análise da Germinação: Um Enfoque Estatístico. Brasília: Ed. Universidade de Brasília, 2004. $248 \mathrm{p}$.

TAO, K. L. J. Physiological rupture of hypocotylis in germination and vigor tests. Journal of Seed Technology, v. 6, n. 3, p. 1-8, 1981.

TOLEDO, F. F.; PEDREIRA, A. A. S. Quantidade de solução de nitrato de potássio e germinação de sementes de capim colonião. Revista Brasileira de Sementes, Pelotas, v. 6, n. 1, p. 61-70, 1984.

VARELA, V. P. et al. Umedecimento do substrato e temperatura na germinação de sementes de angelimpedra (Dinizia excelsa Ducke). Revista Brasileira de Sementes, Pelotas, v. 27, n. 2, p. 130-135, 2005. 